\title{
Overexpression of p21-activated kinase 1 promotes endometrial cancer progression
}

\author{
WEN LU, JUN-JIE QU, BI-LAN LI, CONG LU, QIN YAN, XIAO-MEI WU, \\ XIAO-YU CHEN and XIAO-PING WAN
}

\begin{abstract}
Department of Obstetrics and Gynaecology, The International Peace Maternity \& Child Health Hospital, Shanghai Jiao Tong University, Shanghai, P.R. China
\end{abstract}

Received November 26, 2012; Accepted December 17, 2012

DOI: $10.3892 /$ or.2013.2237

\begin{abstract}
Endometrial cancer (EC) is the most common gynecologic malignancy, but the molecular events involved in the development and progression of EC remain unclear. P21-activated kinase 1 (Pak1) plays important roles in cell motility and survival. This study investigated the clinical significance of Pak1 expression and its functional roles in EC. The expression of Pak1 in clinical samples and EC cell lines was evaluated. The effects of Pak1 on EC cell functions were determined by either overexpressing it via plasmid transfection or depleting its expression using short hairpin RNA (shRNA) in human EC cell lines. Pak1 was overexpressed in clinical samples of EC compared with normal endometrium. High Pak1 expression in EC was positively correlated with lymph node metastasis, advanced disease stage and poor histological differentiation. Pak1 overexpression was also observed in multiple human EC cell lines. In EC cell lines, Pak1 overexpression promoted cell proliferation, migration, invasion and anchorage-independent growth in vitro. Conversely, shRNAmediated stable knockdown of Pak1 reduced cell proliferation, migration, invasion and anchorage-independent growth. In addition, ectopic Pak1 overexpression protected EC cells from apoptosis, along with decreased caspase-3 activation. These results suggest that Pak1 plays important roles at multiple stages of EC progression.
\end{abstract}

Correspondence to: Dr Xiao-Ping Wan, Department of Obstetrics and Gynaecology, The International Peace Maternity \& Child Health Hospital, Shanghai Jiao Tong University, 910 Hengshan Road, Shanghai 200030, P.R. China

E-mail: wanxp@sjtu.edu.cn

Abbreviations: BAD, Bcl-2-associated death promoter; BCL2, B-cell lymphoma 2; DLC1, dynein light chain 1; EC, endometrial cancer; ER $\alpha$, estrogen receptor $\alpha$; MAPK, mitogen-activated protein kinase; NSCLC, non-small cell lung cancer; Pak1, p21-activated kinase 1; qRT-PCR, quantitative reverse transcription-polymerase chain reaction; TNF- $\alpha$, tumor necrosis factor $\alpha$

Key words: p21-activated kinase 1, endometrial cancer, metastasis, apoptosis, caspase-3

\section{Introduction}

Endometrial carcinoma is the most common malignancy of the female genital tract, with an estimated 46,470 new cases and 8,120 deaths in the United States in 2011 (1). In other countries, such as China, its incidence is on the rise (2). For young women who wish to retain their fertility, only 50\% show a complete response to hormonal treatment (usually progestins), while $25 \%$ suffer a relapse after a temporary response (3). Patients with metastatic and refractory cancer are at a significantly higher risk of morbidity and mortality, with a median survival of 8-16 months (4). The poor prognosis is attributed to the significant failure rate of traditional adjuvant therapy, including radiotherapy, chemotherapy and hormonal therapy, following tumor debulking (5). To improve the outcome of patients with endometrial carcinoma, it is important to investigate molecular pathways that are critical to the development of the disease, and to identify novel targets for therapy.

The p21-activated kinases (Paks) are a family of conserved non-receptor serine/threonine kinases that integrate various signaling pathways that are vital to normal cell survival and function (6). Based on their sequence and functional similarities, six mammalian Paks are subdivided into two groups: group I (pak1-3) and group II (pak4-6) (7). Pak1 was originally identified as a downstream effector of the Rho family small GTPases Cdc42 and Rac1, which play a fundamental role in cytoskeleton reorganization and motility $(8,9)$. Subsequent studies have revealed that Pak1 is also involved in regulating cell growth (10), apoptosis (11), adhesion (12) and angiogenesis (13), all of which are important for tumorigenesis and metastasis.

Pak1 is widely expressed in a variety of normal tissues, such as brain, muscle and spleen (14). Previous studies indicated that Pak1 expression is significantly increased in breast, colorectal and ovarian cancer (15-17). Functional studies have also implicated Pak1 in cell transformation and tumor cell invasion (14). For instance, Pak1 has been shown to promote non-small cell lung cancer (NSCLC) cell motility and invasiveness by phosphorylation of CRK-II (c-Crk) (18). In addition, Pak1 was able to promote mammary epithelial hyperplasia through phosphorylation and transactivation of estrogen receptor- $\alpha$ $(E R \alpha)(10)$, which is also involved in the pathogenesis of endometrial cancer (EC) (19). However, the expression pattern and the function of Pak1 in EC remain unknown. 
In this study, we sought to determine whether Pak1 is involved in the pathogenesis of EC. The expression of Pak1 in EC cell lines and tissues was assessed and correlated with clinicopathological parameters. We also investigated the effect of Pak1 on EC cell proliferation, migration, invasion, anchorageindependent growth and survival. Our results indicate that Pak1 is a multifunctional regulator of the progression of EC.

\section{Materials and methods}

Clinical samples. A total of 42 paraffin embedded EC tissues (including 10 metastasis-positive lymph nodes) were retrieved from 32 patients with endometrial carcinoma at the Department of Pathology at the International Peace Maternity and Child Health Hospital, affiliated to Shanghai Jiao Tong University, China, from December 2009 to November 2011. The diagnosis of each sample was established by two pathologists according to the criteria of the International Federation of Gynecology and Obstetrics (FIGO). These patients ranged in age from 44 to 78 years (mean, 59 years). None had hormone therapy, radiotherapy, or chemotherapy prior to surgery.

Twenty-three normal endometrial samples were obtained from patients who underwent a hysterectomy to treat other diseases, such as myoma or adenomyosis. These patients ranged in age from 27 to 64 years (mean, 46 years). Ten endometrial hyperplasia tissues (5 without atypical cases and 5 with atypical cases) were collected from patients who had undergone hysteroscope examination due to irregular bleeding. The collection of clinical samples was approved by the Ethics Committee of the Medical Faculty of Shanghai Jiao Tong University, China. All patients provided written informed consent.

Immunohistochemistry analysis. The immunohistochemical staining procedure was as follows: formalin-fixed sections were deparaffinized with xylene and hydrated in alcohol; for antigen retrieval, slides were immersed in citrate buffer (pH 6.0) and boiled for $20 \mathrm{~min}$. These sections were then incubated in $0.3 \% \mathrm{H}_{2} \mathrm{O}_{2}$ in methanol for $30 \mathrm{~min}$ to inactivate endogenous peroxidase activity. Non-specific reaction was blocked by incubating slides with $5 \%$ bovine serum albumin for $30 \mathrm{~min}$ at room temperature. Sections were incubated overnight at $4^{\circ} \mathrm{C}$ with a 1:100 dilution of rabbit anti-Pak 1 antibody (Abcam, Cambridge, MA, USA). Preimmune serum from the same species was used as a negative control. Subsequently, slides were incubated with biotinylated secondary antibody (Mrbiotech, Emeryville, CA, USA) for $10 \mathrm{~min}$.

The staining intensity was scored in the following manner: 0 , negative; 1 , weak; 2 , moderate; 3 , strong. The staining percentage was scored as: $0,0-5 \% ; 1,5-25 \% ; 2,25-50 \%$; 3,50-75\%; and 4, 75-100\%. We obtained a composite histoscore by multiplying the values of the 2 parameters by a maximum of $12(20)$.

Cell culture and isolation of human endometrial epithelial cells. Four EC cell lines were purchased from ATCC (American Type Culture Collection, Rockville, MD, USA). All cell lines were cultured in DMEM/F12 (1:1) (Gibco, Auckland, New Zealand) and supplemented with $10 \%$ fetal bovine serum (FBS) (HyClone, Logan, UT, USA) in a $37^{\circ} \mathrm{C}$, $5 \% \mathrm{CO}_{2}$ incubator.
Primary human endometrial epithelial cells were cultured as described by Zhang et al (21) and Osteen et al (22). Briefly, the normal endometrial tissue was minced into small pieces and digested with $0.1 \%$ trypsin-EDTA for $30 \mathrm{~min}$ at $37^{\circ} \mathrm{C}$. Using serial filtration, tissue debris was separated with $150-\mu \mathrm{m}$ aperture sieves. Epithelial glands were retained on $40-\mu \mathrm{m}$ aperture sieves and backwashed with PBS. The separated endometrial cells were cultured in DMEM/F12 (1:1) supplemented with $10 \%$ FBS. All specimens were confirmed as normal by histological analysis.

Plasmidandtransfection. To stably express Pak1 in AN3CA and Ishikawa ells, the cells were transfected with Pak1 expressing vector or the empty control vector pEGFP-N1 (Clontech Laboratories, Palo Alto, CA, USA) using Lipofectamine 2000 (Invitrogen, Carlsbad, CA, USA), and then selected with G418 (800 $\mu \mathrm{g} / \mathrm{ml}$; Sigma Chemical, St. Louis, MO, USA). To stably silence Pak1, the cells were transfected with a set of shRNA constructs (GeneCopoeia, Germantown, MD, USA) against human Pak1 or the scrambled control vector psiHIV-U6 (GeneCopoeia), and then selected with puromycin $(0.5 \mu \mathrm{g} / \mathrm{ml}$; Sigma Chemical).

Real-time $q R T$-PCR. Total RNA was extracted from cells using TRIzol reagent (Invitrogen). cDNA was reverse-transcribed from total RNA using Prime Script RT reagent kit (Takara Inc., Otsu, Japan). Real-time PCR was performed using SYBR Premix Ex Taq (Takara Inc.) and analyzed with an ABI Prism 7000 Sequence Detection System. The oligonucleotide primers used included: Pak1 forward: 5'-AGTTTCAGAAGATGAG GATGATGA-3', reverse: 5'-AATCACAGACCGTGTGTAT ACAG-3'; and $\beta$-actin forward: 5'-CAGCCATGTACGTTG CTATCCAGG-3', reverse: 5'-AGGTCCAGACGCAGGATG GCATG-3'. Relative transcript expression levels were calculated using the $2^{-\Delta \Delta \mathrm{C}_{\mathrm{T}}}$ method using $\beta$-actin as internal reference. All data were obtained in triplicate in three independent experiments.

Western blot analysis. Cells were harvested with ProteoJET Mammalian Cell Lysis Reagent (MBI Fermentas, Burlington, ON, Canada) with a protease inhibitor cocktail (Roche Diagnostics, Basel, Switzerland). A total of $60 \mu \mathrm{g}$ protein was separated by SDS-PAGE, and transferred to a polyvinylidene fluoride (PVDF) membrane. Membranes were incubated with primary antibodies. Signal was detected using BeyoECL Plus (Beyotime, Shanghai, China). Primary antibodies included: rabbit anti-Pak1 (1:1000; Abcam), mouse anti- $\beta$-actin (1:2000; ProteinTech Group, Chicago, IL, USA).

Proliferation assays. Cells $\left(2 \times 10^{3}\right.$ cells/well) were plated in 96 -well plates. Cell number was measured every $24 \mathrm{~h}$ by MTT assay following the manufacturer's instructions (Beyotime). The OD value of each well was detected at $570 \mathrm{~nm}$. Medium was changed every other day. Each experiment was repeated in triplicate.

In vitro migration and invasion assays. Cell migration and invasion assays were performed using culture medium-treated 6.5-mm transwell chambers with $8.0 \mu \mathrm{m}$ polycarbonate membranes (Corning LifeScience, Corning, NY, USA). 
A

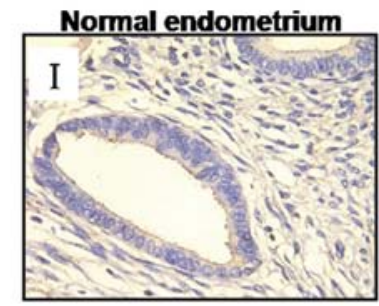

Serous carcinoma

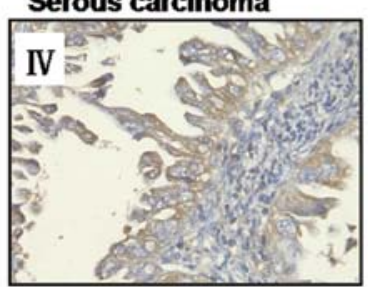

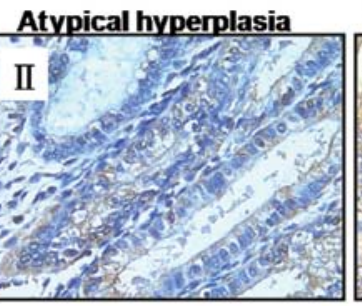

Clear cell carcinoma

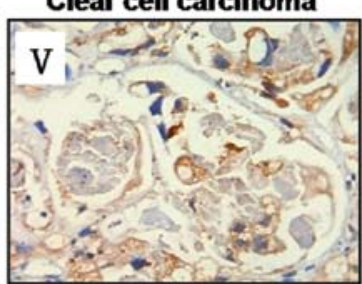

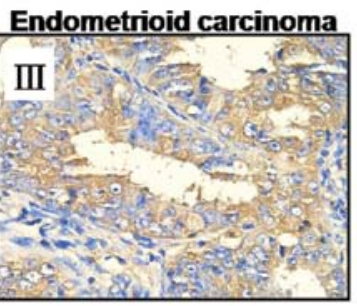

Lymph node metastasis

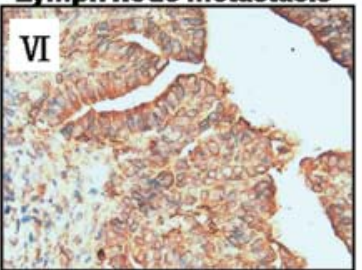

B

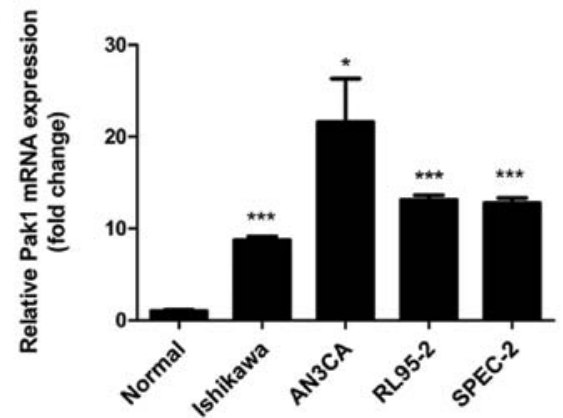

C

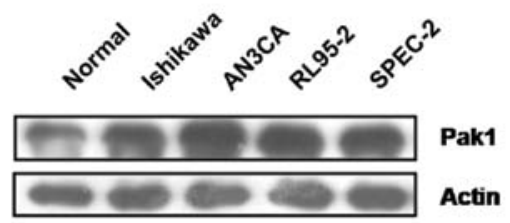

Figure 1. Pak1 is overexpressed in endometrial cancer. (A) Pak1 immunohistochemical staining in normal endometrium, atypical hyperplasia, endometrioid carcinoma, serous carcinoma, clear cell carcinoma and lymph node metastasis. Original magnification, $\mathrm{x} 400$. (B) mRNA and (C) protein expression of Pak1 in primary cultured normal endometrial epithelial cells and endometrial cancer cell lines as determined by $\mathrm{qRT}-\mathrm{PCR}\left({ }^{*} \mathrm{P}<0.05\right.$, ${ }^{* * *} \mathrm{P}<0.001$ compared with normal) and western blot analysis, respectively.

Matrigel (BD Biosciences, San Jose, CA, USA) coated transwell membrane was used for invasion assay. Cells were seeded in serum-free medium on the upper compartment of each transwell chamber at a density between $2 \times 10^{5}-1 \times 10^{6}$ cells $/ \mathrm{ml}$ (200 $\mu \mathrm{l} /$ chamber). The lower chamber was filled with culture medium containing $10 \%$ FBS. After $24 \mathrm{~h}$ of incubation, non-migrated or non-invaded cells were removed from the upper chamber. The invaded or migrated cells on the lower side of inserts were fixed and stained with $5 \%$ crystal violet. Attached cells were lysed with $10 \%$ ethylic acid, and absorbance (proportional to the number of cells) was measured at $590 \mathrm{~nm}$.

Soft agar colony assays. Cells were seeded in $0.3 \%$ top agar in growth medium over a layer of $0.6 \%$ agar in a 6 -well plate at a density of $1 \times 10^{4}$ cells $/ \mathrm{ml}$. After 3 weeks of incubation, colonies of $>50$ cells were produced. Only colonies with $>50$ cells were counted and photographed with an inverted microscope. All assays were performed at least three times in triplicate.

Hoechst staining. Cells were seeded in 24-well plates and treated with TNF- $\alpha$ (20 ng/ml; Peprotech, Rocky Hill, NJ, USA). At the indicated time point, cells were washed in PBS and fixed in $4 \%$ (wt/vol) paraformaldehyde for $16 \mathrm{~h}$ at $4^{\circ} \mathrm{C}$. Cells were washed in PBS and permeabilized with $0.2 \%$ Triton $\mathrm{X}-100$ for $20 \mathrm{~min}$ at room temperature. Permeabilized cells were then treated with Hoechst $33258(10 \mathrm{mg} / \mathrm{ml})$ for $30 \mathrm{~min}$ at room temperature. Photomicrographs of cells were captured under fluorescence at a magnification of $\mathrm{x} 400$.

Flow cytometry. Annexin V-TRITC apoptosis detection kit (KeyGen Biotech, Shanghai, China) was used to identify the percentage of apoptosis, following the manufacturer's instructions. Briefly, after stimulation, both floating and attached cells were collected by low speed centrifugation. After washing in PBS, cells were stained with Annexin V-TRITC and 7-AAD. Following incubation for $30 \mathrm{~min}$ at room temperature, the cells were analyzed by flow cytometry (Becton Dickinson FACScan, Immunocytochemistry Systems, San Jose, CA, USA). Tests were performed in triplicate.

Caspase activity assay. The enzymatic activity of caspase-3 was measured according to the manufacturer's instructions (Caspase-3 Activity Assay kit, Beyotime). After 0-12 h of TNF- $\alpha$ exposure, $2 \times 10^{6}$ cells were collected and suspended in cell lysis buffer. Cell lysates were mixed with reaction buffer at $37^{\circ} \mathrm{C}$ for $1 \mathrm{~h}$. An increase in absorbance at $405 \mathrm{~nm}$ was used to quantify the activation of caspase- 3 activity. Caspase activity was expressed as percentage of enzyme activity compared to control. Control groups received 0.1\% DMSO. All the experiments were carried out in triplicate.

Statistical analysis. All tests were carried out with SPSS 16.0 (Microsoft, Redmond, WA, USA) or Prism (GraphPad, 
Table I. Correlation of Pak1 expression with clinicopathological parameters in endometrial carcinomas.

\begin{tabular}{|c|c|c|c|}
\hline \multirow[b]{2}{*}{ Characteristics } & \multirow[b]{2}{*}{ Case (n) } & \multicolumn{2}{|c|}{ Pak1 histoscore } \\
\hline & & Mean \pm SD & P-value \\
\hline \multicolumn{4}{|l|}{ Diagnostic categories } \\
\hline Normal & 23 & $4.70 \pm 2.23$ & \\
\hline Hyperplasia & 10 & $6.70 \pm 1.50$ & \\
\hline Carcinomas & 32 & $9.63 \pm 2.85$ & \\
\hline Lymph node metastasis ${ }^{c}$ & 10 & $12.00 \pm 0.00$ & $\leq 0.001^{\mathrm{a}}$ \\
\hline \multicolumn{4}{|l|}{ Age } \\
\hline$<60$ & 19 & $9.58 \pm 2.80$ & \\
\hline$\geq 60$ & 13 & $9.69 \pm 3.04$ & $0.966^{\mathrm{b}}$ \\
\hline \multicolumn{4}{|l|}{ Histology } \\
\hline Endometrioid & 24 & $9.00 \pm 2.95$ & \\
\hline Non-endometrioid (serous/clear) & 8 & $11.50 \pm 1.41$ & $0.051^{\mathrm{b}}$ \\
\hline \multicolumn{4}{|l|}{ FIGO stage } \\
\hline Early (I-II) & 24 & $8.83 \pm 2.88$ & \\
\hline Late (III-IV) & 8 & $12.00 \pm 0.00$ & $\underline{0.013}^{\mathrm{b}}$ \\
\hline \multicolumn{4}{|l|}{ Histological grade } \\
\hline Low $(1-2)$ & 16 & $7.75 \pm 2.72$ & \\
\hline High (3) & 16 & $11.50 \pm 1.37$ & $\leq 0.001^{\mathrm{b}}$ \\
\hline \multicolumn{4}{|l|}{ Myometrial invasion } \\
\hline$\leq 1 / 2$ & 27 & $9.19 \pm 2.90$ & \\
\hline$>1 / 2$ & 5 & $12.00 \pm 0.00$ & $\underline{0.043}^{\mathrm{b}}$ \\
\hline \multicolumn{4}{|l|}{ Involving cervix } \\
\hline Negative & 27 & $9.19 \pm 2.90$ & \\
\hline Positive & 5 & $11.20 \pm 1.79$ & $\underline{0.043}^{\mathrm{b}}$ \\
\hline \multicolumn{4}{|l|}{ Vascular space invasion } \\
\hline Negative & 18 & $8.44 \pm 3.03$ & \\
\hline Positive & 14 & $11.14 \pm 1.70$ & $\underline{0.014^{\mathrm{b}}}$ \\
\hline
\end{tabular}

Significance of difference (P-value) between categories was analyzed by ${ }^{\mathrm{a} K r u s k a l-W a l l i s ~ r a n k ~ t e s t ~ a n d ~}{ }^{\mathrm{b}} \mathrm{Mann}-\mathrm{Whitney} \mathrm{U}$ test, respectively. Those with significant $\mathrm{P}$-values are underlined. ${ }^{\mathrm{c}} \mathrm{A}$ total of 10 specimens from eight patients.

San Diego, CA, USA). Each experiment was performed at least three times. Where applicable, data are shown as the means \pm SD. The two-tailed Student's t-test or Mann-Whitney $\mathrm{U}$ test were used for comparison of data between two groups. Differences were considered statistically significant at $\mathrm{P}<0.05$.

\section{Results}

Pakl is overexpressed in endometrial cancer tissues and cell lines. Immunochemistry staining showed that Pak1 protein was predominantly localized to the cytoplasm of endometrial epithelial cells. There was only weak or no staining in normal endometrium, whereas moderate to strong Pak1 immunostaining was found in endometrial hyperplasia and endometrial carcinoma tissues. The most intense staining was found in lymph node metastases (Fig. 1A).

To account for both stain intensity and the uniformity of stain, a composite histoscore (percentage epithelium stained $\mathrm{x}$ stain intensity) was calculated. Analyzed by the Kruskal-Wallis rank test, cytoplasmic Pak1 expression (Pak1 composite histoscore) progressively increased in the epithelial cells of endometrial hyperplasia, invasive carcinomas, and lymph node metastases relative to the normal endometrium $(\mathrm{P}<0.001$; Table I).

We next explored the correlation of Pak1 expression levels with clinicopathological parameters in endometrial carcinomas. Significantly higher cytoplasmic Pak1 expression was found in carcinomas of advanced stage (stages III and IV) and poor histological differentiation (grade 3) (all $\mathrm{P}<0.05$; Table I). Additionally, increased Pak1 expression was significantly associated with depth of myometrial invasion, cervix involvement and vascular space invasion (all $\mathrm{P}<0.05$; Table I). However, there was no significant difference between endometrioid and non-endometrioid (serous and clear cell histological subtypes) endometrial carcinomas $(\mathrm{P}=0.051$; Table I). 
A

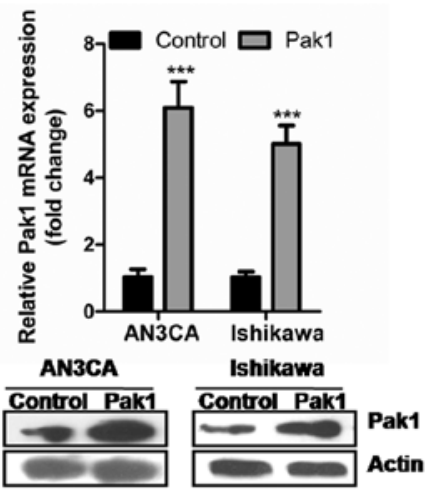

D

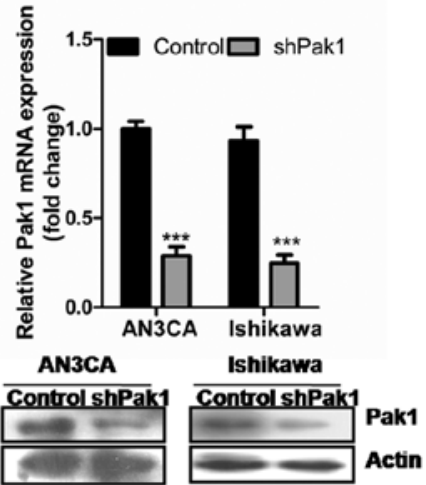

B

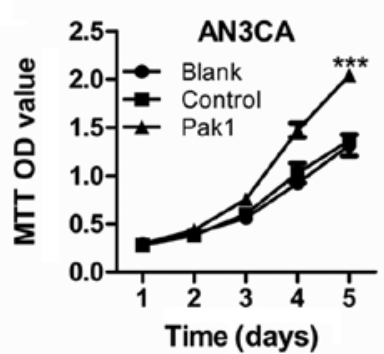

E

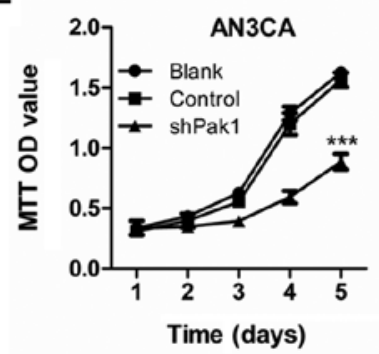

C

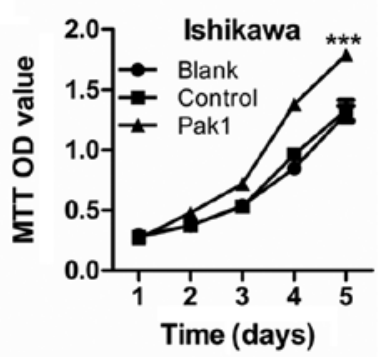

F

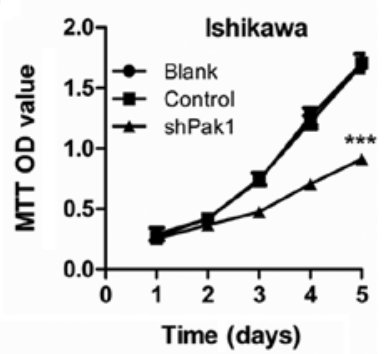

Figure 2. Pak1 promotes endometrial cancer cell proliferation. (A) mRNA and protein expression of Pak1 were measured in Pak1 stable overexpression AN3CA and Ishikawa cells by qRT-PCR (upper) and western blot analysis (lower), respectively; ${ }^{* * *} \mathrm{P}<0.001$. (B and C) MTT assay applied for cellular proliferation in AN3CA and Ishikawa cells stably transfected with Pak1 expression vector (Pak1) or control vector (control) or non-transfected cells (blank); ${ }^{* * *} \mathrm{P}<0.001$. (D) shRNA effectiveness was confirmed by significantly decreased Pak1 mRNA (upper) and protein (lower) expression in AN3CA and Ishikawa cells; ${ }^{* * *} \mathrm{P}<0.001$. (E and F) Cell proliferation was significantly reduced in Pak1 stable knockdown AN3CA and Ishikawa cells compared to scrambled controls; ${ }^{* * *} \mathrm{P}<0.001$

Moreover, using real-time qRT-PCR and immunoblotting, we detected higher levels of Pak1 mRNA (Fig. 1B) and protein (Fig. 1C) in EC cell lines compared with primary cultured normal endometrial epithelial cells. The elevated Pak1 expression in endometrial carcinoma determined above suggests that overexpression of Pak1 might contribute to the malignant progression of human EC.

Pakl promotes endometrial cancer cell proliferation. Immunohistochemical staining showed that Pak1 expression was associated with the progression of EC. To investigate the potential role of Pak1 in EC cell proliferation, AN3CA and Ishikawa cells were stably transfected with Pak1 expressing vector or empty control vector (Fig. 2A). Proliferation assays revealed that stable overexpression of Pak1 significantly induced AN3CA and Ishikawa cell growth (Fig. 2B and C) $(\mathrm{P}<0.001, \mathrm{P}<0.001)$.

To further investigate the effect of Pak1 on EC cell proliferation, we performed short hairpin RNAs (shRNA)-mediated stable knockdown of Pak1 in AN3CA and Ishikawa cells. As shown in Fig. 2D, the shRNAs led to an almost complete loss of Pak1 in EC cell lines compared to the scrambled control cells. Significantly reduced cell proliferation was observed after Pak1 stable knockdown (Fig. 2E and F) $(\mathrm{P}<0.001, \mathrm{P}<0.001)$.

Pakl promotes endometrial cancer cell migration and invasion. We next examined the effect of Pak1 on EC cell migration and invasion. The transwell migration and invasion assays were performed to study the migratory and invasive ability of EC cells. As shown in Fig. 3A and B, stable overexpression of Pak1 in AN3CA and Ishikawa cells significantly increased cell migration and invasion compared with cells transfected with control vector $(\mathrm{P}<0.001, \mathrm{P}<0.001)$. Conversely, shRNAmediated knockdown of Pak1 in AN3CA and Ishikawa cells resulted in significantly decreased cell migration and invasion. Cells transfected with shRNA expression vector showed $>50 \%$ defective migration and invasion compared with cells transfected with scrambled control vector (Fig. 3C and D) (P<0.001, $\mathrm{P}<0.001)$. These results indicate that Pak1 is an important participant in EC cell invasion and metastasis.

Pakl promotes endometrial cancer cell anchorage-independent growth. We next investigated whether Pak1 is required in anchorage-independent growth, a hallmark of oncogenic transformation (23). The soft agar assays were performed to test the transformation of EC cells (24). Pak1 expressing vector and control vector transfected EC cells were plated in soft agar and scored for colony formation after 3 weeks. As shown in Fig. 4A, there was a significant difference in the number and size of colonies between vector-transfected control cells and Pak1 overexpression AN3CA and Ishikawa cells $(\mathrm{P}<0.001$, $\mathrm{P}<0.01)$. By contrast, stable knockdown of Pak1 almost completely abolished colony formation of EC cells in soft agar (Fig. 4B). These findings indicate that Pak1 promoted EC cell anchorage-independent growth. 
A
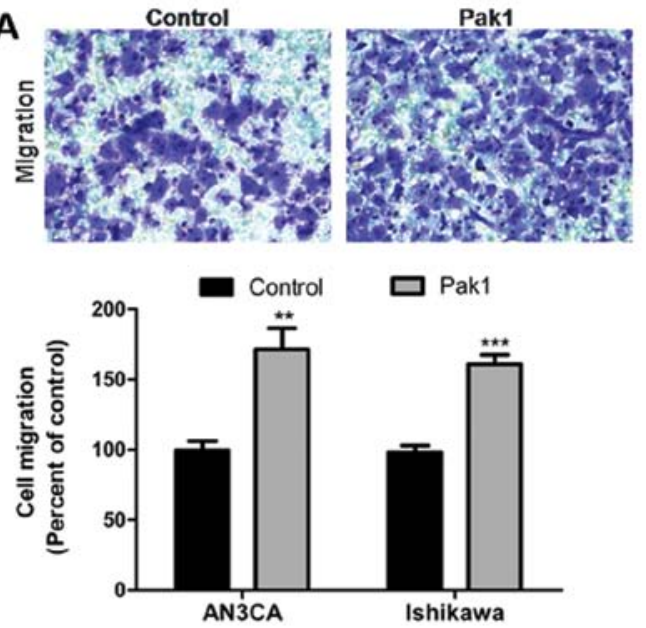

C
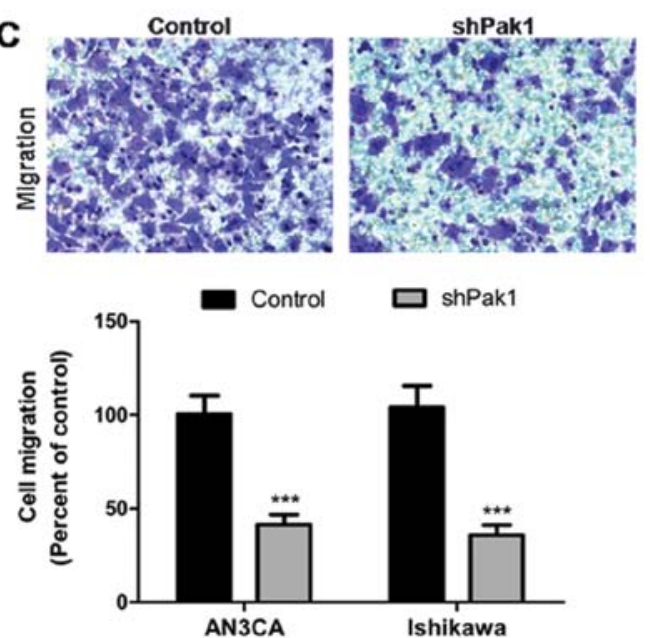
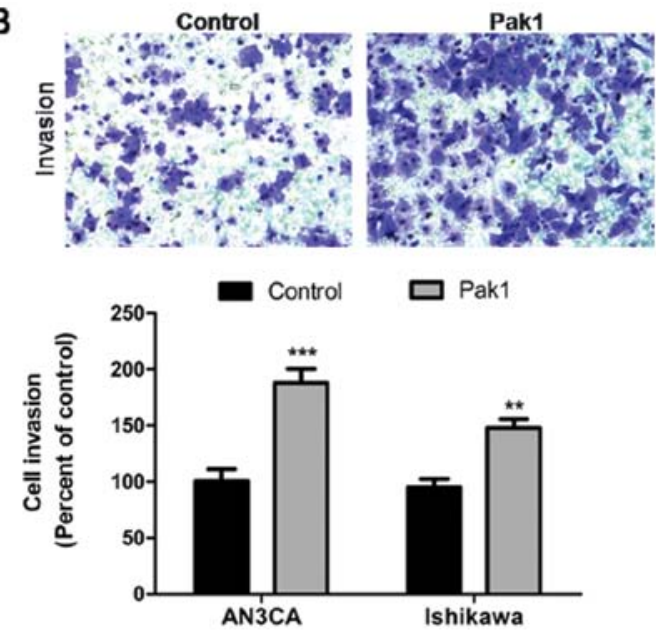

D
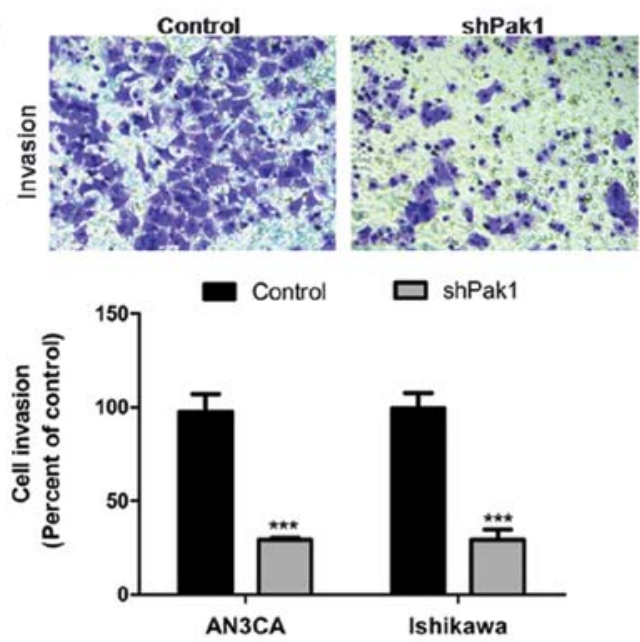

Figure 3. Pak1 promotes endometrial cancer cell migration and invasion. In vitro migration (A) and invasion (B) assays using Transwell membrane without or with Matrigel coating, respectively. AN3CA and Ishikawa cells were stably transfected with Pak1 or control vector; (upper) representative images of migrating or invading EC cells are shown (magnification, x200). Cell migration or invasion of EC cells presented as percentage of control (lower), OD values of permeated cells eluted in $10 \%$ ethylic acid elution were compared; $n=3 ;{ }^{* *} \mathrm{P}<0.01,{ }^{* * * *} \mathrm{P}<0.001$. (C and $\mathrm{D}$ ) Cell migration and invasion were significantly reduced in Pak1 stable knockdown AN3CA and Ishikawa cells compared to scrambled controls; ${ }^{* * *} \mathrm{P}<0.001$.

Pakl protects endometrial cancer cells from apoptosis via inhibition of caspase-3 activation. Since overexpression of Pak1 results in increased growth of EC cells, we sought to determine whether Pak1 also plays a protective role against apoptosis in EC cells. To elucidate this possibility, vector control cells and Pak1 overexpressing EC cells were treated with TNF- $\alpha$. As shown in Fig. 5A, at 2 h, more control cells had condensed nuclei compared to Pak1 overexpressing cells, as determined by Hoechst staining. At $12 \mathrm{~h}$, flow cytometry using Annexin $\mathrm{V}$ and 7-AAD showed that a higher percentage of apoptotic cells were observed in control cells compared with Pak1 overexpressing cells (Fig. 5B and C). To further elucidate the mechanism by which Pak1 acts, we tested the activation of caspase-3, a well known downstream target of the TNF- $\alpha$ induced death pathway (25). We found that overexpression of Pak1 markedly abolished TNF- $\alpha$ induced caspase-3 activation (Fig. 5D). Our results indicate that, at least in response to TNF- $\alpha$, Pak1 plays an essential role in protecting cells from apoptosis via inhibition of caspase-3 activation.

\section{Discussion}

Pak1 is upregulated and activated in several human tumor types (6). However, the expression of Pak1 in EC tissue and its relationship with pathological parameters remains unknown. In the present study, significantly increased Pak1 protein expression in clinical EC samples and cell lines was detected relative to normal endometrial tissues and primary cultured normal endometrial epithelial cells. Furthermore, Pak1 protein expression is additionally increased in lymph node metastases. These data indicate that Pak1 expression is specifically increased in the most advanced lesions of EC. We found a significantly positive correlation between Pak1 expression with advanced disease stage and poor histological differentiation, which further suggests that Pak1 expression is increased with EC progression. Collectively, these data indicate that overexpression of Pak1 might contribute to malignant progression of human EC.

Pak1 has been shown to be involved in the rapid proliferation of cancer cells $(17,26)$. Pak1 promotes proliferation 
A

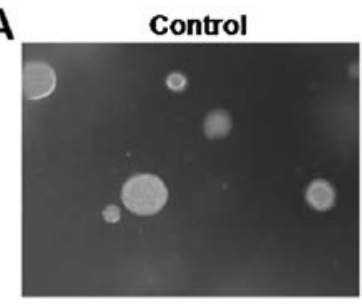

B

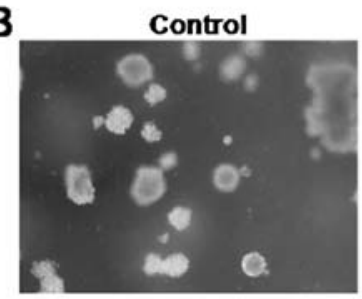

Pak1

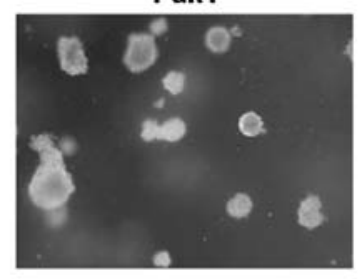

shPak1

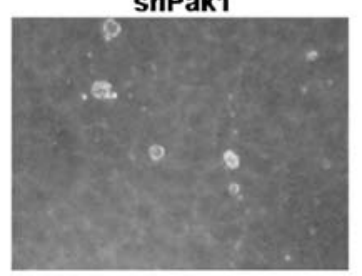

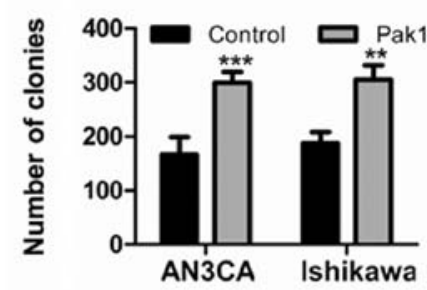

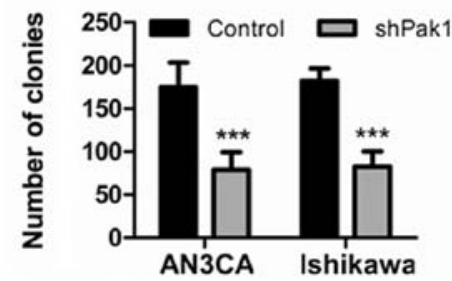

Figure 4. Pak1 promotes EC cell anchorage-independent growth. (A) Stable EC cell lines expressing Pak1 were assayed for anchorage-independent growth in soft agar. AN3CA and Ishikawa cells that had been stably transfected with the empty vector were used as controls. Colony formation ( $\geq 50$ cells) was assessed using a colony counter. (B) Knockdown of Pak1 led to a significant decrease in the colony-forming ability of EC cells. Representative images (left) were captured with an inverted phase contrast microscope (magnification, x200). Columns (right), mean number of colonies from three independent experiments, each in triplicate; bars, $\mathrm{SD} ;{ }^{* *} \mathrm{P}<0.01,{ }^{* * *} \mathrm{P}<0.001$.
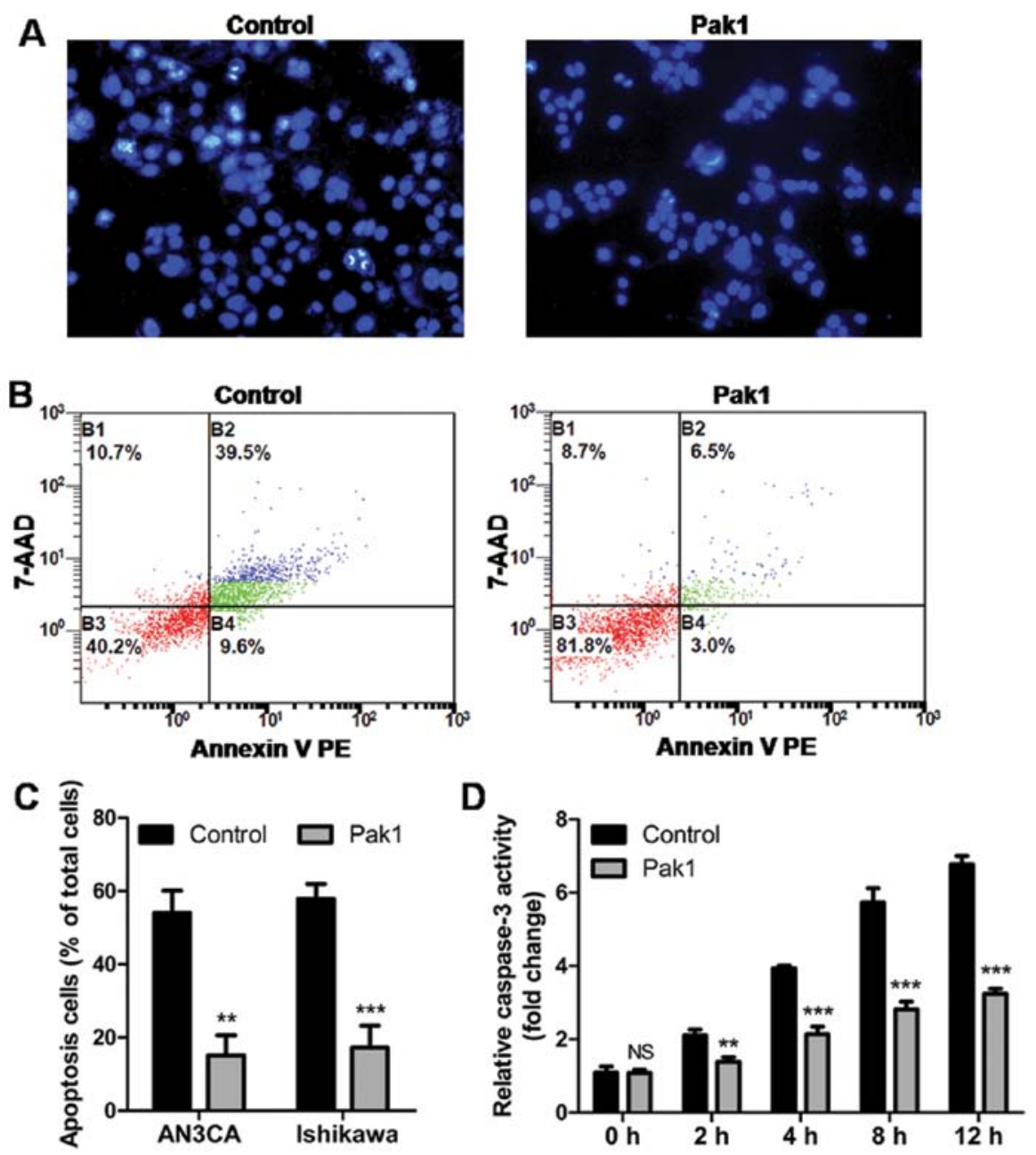

Figure 5. Pak1 prevents caspase-3 activation and protects cells from TNF- $\alpha$ induced apoptosis. (A) Nuclear condensation after $2 \mathrm{~h}$ of TNF- $\alpha$ (20 ng/ml) treatment was detected by fluorescence microscopy analysis of control and stable Pak1-expressing EC cells stained with Hoechst 33258. Photographed representative numbers of apoptotic cells are shown. (B) FACS analysis for Annexin V and 7-AAD staining in control and stable Pak1-expressing EC cells. (C) Control and stable Pak1-expressing EC cells were treated with $20 \mathrm{ng} / \mathrm{ml} \mathrm{TNF-} \alpha$, and cells were harvested at the indicated number of hours. (D) Caspase-3 activity was expressed as percentage of enzyme activity compared to $0 \mathrm{~h} ;{ }^{* *} \mathrm{P}<0.01,{ }^{* * *} \mathrm{P}<0.001$. All the experiments were carried out in triplicate. 
through the NF- $\kappa \mathrm{B}$-dependent pathway that regulates cyclin D1 transcription in breast cancer (17). Knockdown of Pak1 in colorectal cancer cells decreased proliferation and delayed the G1/S cell-cycle transition (26). In this study, we were able to confirm effect of Pak1 on proliferation from two aspects: Pak1 ectopic expression significantly enhanced proliferation whereas shRNA-mediated knockdown of Pak1 efficiently impeded proliferation in EC cells. Pak1 is a common point of convergence of growth factor signaling (27), and also directly interacts with estrogen receptor (ER) in ER-positive breast cancer (28). It has been proposed that Pak1 may constitute an important point of cross-talk between growth factors and the ER (29). Crosstalk between ER and growth factor signaling has emerged as a critical factor in endocrine resistance in EC (30). If this is the case, the levels and activation of Pak1 could potentially affect the action of anti-estrogen therapies. In experimental breast cancer model systems, overexpression of Pak1 correlates with the development of ligand-independent stimulation of ER $\alpha$ signaling, which is associated with sensitivity to tamoxifen (a selective anti-estrogen, which has anti-estrogenic effects in the breast but weak estrogenic effects in the endometrium) $(28,31)$. Therapies that target Pak1 may therefore represent a strategy to increase the hormonal treatment response in EC.

We further investigated the effect of Pak1 on enhancing cell migration and invasion of EC cell lines. Tumor cell migration and invasion requires coordinated reorganization of the actin cytoskeleton (32). Pak1 is thought to regulate actin reorganization through several reported substrates (6). For example, Pak1 phosphorylates LIM-kinase at threonine 508 within kinase's activation loop, and increases the phosphorylation and activation of cofilin, thus regulating actin depolymerization (9). Inhibition of Pak1 abolishes filamentous actin (F-actin) flow in the lamella, displaces myosin IIA from the cell edge, and decreases focal adhesion turnover (33). Pak1-knockdown cells have significantly impaired migration and invasion $(16,34,35)$. In the present study, we also showed that downregulation of Pak1 expression reduced migration and invasion of EC cells.

Anchorage-independent growth is an important hallmark of oncogenic transformation (23). The expression of kinase-active Pak1 mutants significantly stimulates anchorageindependent growth of breast cancer cells in soft agar in a preferential mitogen-activated protein kinase (MAPK)sensitive manner (36). Pak1 also phosphorylates dynein light chain 1 (DLC1) on serine 88 , and promotes breast cancer cell anchorage-independent growth and tumor formation (37). In addition, Pak1 simultaneously activates MAPK and MET signaling, that permit human mammary epithelial cells to form anchorage-independent colonies (38). Herein, we showed that knockdown of Pak1 reduces, and overexpression enhances, the colony-forming ability of EC cells. These results demonstrate the ability of Pak1 expression to stimulate the growth of EC cells in an anchorage-independent manner, and indicate that Pak1 may play an essential role in oncogenic transformation.

The efficacy of cancer treatments depends not only on the cellular damage they cause but also on the cell's ability to respond to these damages by inducing the apoptotic response (5). Pak1 has been shown to be involved in the regulation of several important pro-apoptotic pathways (6). For instance, Pak1 has been reported to directly phosphorylate and inactivate the pro-apoptotic functions of B-cell lymphoma 2
(BCL2) antagonist of cell death (BAD), which results in cell survival (39). In our study, Pak1 was found to protect EC cells from apoptosis induced by TNF- $\alpha$ via inhibition of caspase- 3 activation. Alteration of this signal transduction pathway leading to apoptosis has been reported in drug-resistant cells (5). For instance, reduced caspase-3 activity is associated with cisplatin resistance in human ovarian cancer (40). In human bladder carcinoma, activation of AKT increases paclitaxel resistance by increasing Bad phosphorylation, leading to decreased release of mitochondrial cytochrome $c$ and caspase-3-mediated apoptosis (41). In EC, caspase-3 expression is associated with patient survival (42). We found that expression of Pak1 leads to an inhibition of caspase-3 activation, which might be associated with cisplatin and/or paclitaxel resistance in EC. A recent study showed that dual inhibition of Pak1 and inhibitor of apoptosis proteins (IAPs) efficiently increased effector caspase activation and apoptosis of NSCLC cells (11). Thus, inhibition of Pak1 to enhance chemosensitivity of EC may provide a promising therapeutic option to putative targeted therapy. However, whether Pak1 is involved in protecting cancer cells from apoptosis induced by chemotherapy remains to be determined.

In summary, to the best of our knowledge, we showed for the first time that overexpression of Pak1 plays important roles at multiple stages of EC progression. Our results showed that Pak1 protein is overexpressed in endometrial carcinoma, particularly in the most advanced lesions. We also found that Pak1 affects EC cell proliferation, migration, invasion and anchorage-independent growth in vitro. In addition, Pak1 regulates cell apoptosis through inhibition of caspase-3 activation. Our study demonstrated that Pak1 is a multifunctional regulator of EC, and supports further characterization of Pak1 as a therapeutic target.

\section{Acknowledgements}

This study was supported by Grants from the National Natural Science Funds of China (nos. 81072139, 81172476 and 81272885). The authors thank Professor Hui-Juan Zhang and Dr Yuan Liu for pathology revision.

\section{References}

1. Siegel R, Ward E, Brawley O and Jemal A: Cancer statistics, 2011: the impact of eliminating socioeconomic and racial disparities on premature cancer deaths. CA Cancer J Clin 61: 212-236, 2011.

2. Wang D, Zheng W, Wang SM, et al: Estimation of cancer incidence and mortality attributable to overweight, obesity, and physical inactivity in China. Nutr Cancer 64: 48-56, 2012.

3. Chiva L, Lapuente F, Gonzalez-Cortijo L, et al: Sparing fertility in young patients with endometrial cancer. Gynecol Oncol 111: S101-S104, 2008.

4. Dedes KJ, Wetterskog D, Ashworth A, Kaye SB and ReisFilho JS: Emerging therapeutic targets in endometrial cancer. Nat Rev Clin Oncol 8: 261-271, 2011.

5. Chaudhry P and Asselin E: Resistance to chemotherapy and hormone therapy in endometrial cancer. Endocr Relat Cancer 16: 363-380, 2009.

6. Kumar R, Gururaj AE and Barnes CJ: p21-activated kinases in cancer. Nat Rev Cancer 6: 459-471, 2006.

7. Eswaran J, Soundararajan M, Kumar R and Knapp S: UnPAKing the class differences among p21-activated kinases. Trends Biochem Sci 33: 394-403, 2008.

8. Manser E, Leung T, Salihuddin H, Zhao ZS and Lim L: A brain serine/threonine protein kinase activated by $\mathrm{Cdc} 42$ and Rac1. Nature 367: 40-46, 1994. 
9. Edwards DC, Sanders LC, Bokoch GM and Gill GN: Activation of LIM-kinase by Pak1 couples Rac/Cdc42 GTPase signalling to actin cytoskeletal dynamics. Nat Cell Biol 1: 253-259, 1999.

10. Wang RA, Mazumdar A, Vadlamudi RK and Kumar R: P21-activated kinase-1 phosphorylates and transactivates estrogen receptor-alpha and promotes hyperplasia in mammary epithelium. EMBO J 21: 5437-5447, 2002.

11. Ong CC, Jubb AM, Haverty PM, et al: Targeting p21-activated kinase 1 (PAK1) to induce apoptosis of tumor cells. Proc Natl Acad Sci USA 108: 7177-7182, 2011.

12. Zhou H and Kramer RH: Integrin engagement differentially modulates epithelial cell motility by RhoA/ROCK and PAK1. J Biol Chem 280: 10624-10635, 2005.

13. Bagheri-Yarmand R, Vadlamudi RK, Wang RA, Mendelsohn J and Kumar R: Vascular endothelial growth factor up-regulation via p21-activated kinase-1 signaling regulates heregulin-beta1mediated angiogenesis. J Biol Chem 275: 39451-39457, 2000.

14. Vadlamudi RK and Kumar R: P21-activated kinases in human cancer. Cancer Metastasis Rev 22: 385-393, 2003.

15. Carter JH: Pak-1 expression increases with progression of colorectal carcinomas to metastasis. Clin Cancer Res 10 3448-3456, 2004

16. Siu MK, Wong ES, Chan HY, et al: Differential expression and phosphorylation of Pak1 and Pak2 in ovarian cancer: effects on prognosis and cell invasion. Int J Cancer 127: 21-31, 2010.

17. Balasenthil S: p21-activated kinase-1 signaling mediates cyclin D1 expression in mammary epithelial and cancer cells. J Biol Chem 279: 1422-1428, 2003.

18. Rettig M, Trinidad K, Pezeshkpour G, et al: AK1 kinase promotes cell motility and invasiveness through CRK-II serine phosphorylation in non-small cell lung cancer cells. PLoS One 7: e42012, 2012.

19. Di Cristofano A and Ellenson LH: Endometrial carcinoma. Annu Rev Pathol 2: 57-85, 2007.

20. Siu MK, Chan HY, Kong DS, et al: $\mathrm{p} 21$-activated kinase 4 regulates ovarian cancer cell proliferation, migration, and invasion and contributes to poor prognosis in patients. Proc Natl Acad Sci USA 107: 18622-18627, 2010

21. Zhang L, Rees MC and Bicknell R: The isolation and long-term culture of normal human endometrial epithelium and stroma. Expression of mRNAs for angiogenic polypeptides basally and on oestrogen and progesterone challenges. J Cell Sci 108: 323-331, 1995.

22. Osteen KG, Hill GA, Hargrove JT and Gorstein F: Development of a method to isolate and culture highly purified populations of stromal and epithelial cells from human endometrial biopsy specimens. Fertil Steril 52: 965-972, 1989.

23. Stoker M, O'Neill C, Berryman S and Waxman V: Anchorage and growth regulation in normal and virus-transformed cells. Int J Cancer 3: 683-693, 1968

24. Menendez JA, Vellon L, Colomer R and Lupu R: Oleic acid, the main monounsaturated fatty acid of olive oil, suppresses Her-2/neu (erbB-2) expression and synergistically enhances the growth inhibitory effects of trastuzumab (Herceptin) in breas cancer cells with Her-2/neu oncogene amplification. Ann Oncol 16: 359-371, 2005.

25. Janicke RU, Sprengart ML, Wati MR and Porter AG: Caspase-3 is required for DNA fragmentation and morphological changes associated with apoptosis. J Biol Chem 273: 9357-9360, 1998.
26. Qing H, Gong W, Che Y, et al: PAK1-dependent MAPK pathway activation is required for colorectal cancer cell proliferation. Tumour Biol 33: 985-994, 2012.

27. Gururaj AE, Rayala SK and Kumar R: p21-activated kinase signaling in breast cancer. Breast Cancer Res 7: 5-12, 2005.

28. Rayala SK: P21-activated kinase 1 regulation of estrogen receptoralpha activation involves serine 305 activation linked with serine 118 phosphorylation. Cancer Res 66: 1694-1701, 2006.

29. Kumar R: Signaling intricacies take center stage in cancer cells. Cancer Res 65: 2511-2515, 2005.

30. Gururaj AE: Novel mechanisms of resistance to endocrine therapy: genomic and nongenomic considerations. Clin Cancer Res 12: S1001-S1007, 2006.

31. Kok M, Zwart W, Holm C, et al: PKA-induced phosphorylation of ER $\alpha$ at serine 305 and high PAK1 levels is associated with sensitivity to tamoxifen in ER-positive breast cancer. Breast Cancer Res Treat 125: 1-12, 2010.

32. Gimona M: Mechanics and dynamics of the cytoskeleton: a special issue stemming from the 2008 ECF Meeting/FEBS Workshop (Mechanics and Dynamics of the Cytoskeleton) in Potsdam, Germany. Cell Motil Cytoskeleton 66: ii-iii, 2009.

33. Delorme-Walker VD, Peterson JR, Chernoff J, et al: Pak1 regulates focal adhesion strength, myosin IIA distribution, and actin dynamics to optimize cell migration. J Cell Biol 193: 1289-1303, 2011.

34. McCarty SK, Saji M, Zhang X, et al: Group I p21-activated kinases regulate thyroid cancer cell migration and are overexpressed and activated in thyroid cancer invasion. Endocr Relat Cancer 17: 989-999, 2010.

35. Li LH, Luo Q, Zheng MH, et al: P21-activated protein kinase 1 is overexpressed in gastric cancer and induces cancer metastasis. Oncol Rep 27: 1435-1442, 2012.

36. Vadlamudi RK, Adam L, Wang RA, et al: Regulatable expression of $\mathrm{p} 21$-activated kinase-1 promotes anchorage-independent growth and abnormal organization of mitotic spindles in human epithelial breast cancer cells. J Biol Chem 275: 36238-36244, 2000.

37. Vadlamudi RK, Bagheri-Yarmand R, Yang Z, et al: Dynein light chain 1, a p21-activated kinase 1-interacting substrate, promotes cancerous phenotypes. Cancer Cell 5: 575-585, 2004.

38. Shrestha Y, Schafer EJ, Boehm JS, et al: PAK1 is a breast cancer oncogene that coordinately activates MAPK and MET signaling. Oncogene 31: 3397-3408, 2012.

39. Schurmann A, Mooney AF, Sanders LC, et al: p21-activated kinase 1 phosphorylates the death agonist bad and protects cells from apoptosis. Mol Cell Biol 20: 453-461, 2000.

40. Yang X,Zheng F, Xing H, et al: Resistance to chemotherapy-induced apoptosis via decreased caspase-3 activity and overexpression of antiapoptotic proteins in ovarian cancer. J Cancer Res Clin Oncol 130: 423-428, 2004.

41. Szanto A, Bognar Z, Szigeti A, Szabo A, Farkas L and Gallyas F Jr: Critical role of bad phosphorylation by Akt in cytostatic resistance of human bladder cancer cells. Anticancer Res 29: 159-164, 2009.

42. Peiro G, Diebold J, Baretton GB, Kimmig R and Lohrs U: Cellular apoptosis susceptibility gene expression in endometrial carcinoma: correlation with Bcl-2, Bax, and caspase-3 expression and outcome. Int J Gynecol Pathol 20: 359-367, 2001. 